\title{
ESTUDIO DE LOS PARÁMETROS FISICOQUÍMICOS PARA LA FITORREMEDIACIÓN DE CADMIO (II) Y MERCURIO (II) CON LA ESPECIE Eichhornia crassipes (JACINTO DE AGUA)
}

\author{
Víctor Raúl Poma Llantoy ${ }^{*}$, Ana C. Valderrama Negrón ${ }^{1}$
}

\begin{abstract}
RESUMEN
En este trabajo se realizó estudios para medir la capacidad de sorción de los iones metálicos Cd (II) y $\mathrm{Hg}$ (II) de la especie Eichhornia crassipes (jacinto de agua). Este estudio incluye ensayos donde se optimizan concentración de nutrientes, $p \mathrm{H}$ y concentración de iones metálicos, lo que se llevó a cabo a temperatura ambiente y con soluciones acuosas de Cd (II) y $\mathrm{Hg}$ (II), a las que fueron sometidas las muestras de Eichhornia crassipes. Para corroborar la remoción de dichos metales, las soluciones residuales, después de haber sido sometidas con la especie vegetal, fueron tratadas usando el método APHA 3030-e y las muestras de Eichhornia crassipes fueron tratadas usando el método EPA 200.3. La concentración de Cd (II) fue determinada por un equipo ICP-OES y la del $\mathrm{Hg}$ (II), por un equipo de absorción atómica.

Los resultados obtenidos fueron: Dosis óptima $1 \mathrm{~mL}$ de $\mathrm{A}$ y $0,5 \mathrm{~mL}$ de $\mathrm{B}, p \mathrm{H}$ óptimo 5 , concentración óptima de Cd (II) y Hg (II) $5 \mathrm{mg} / \mathrm{L}$ para cada ion. Con estos parámetros se inició la remoción de $5 \mathrm{mg} / \mathrm{L}$ de los iones metálicos contenidos en 1 litro de solución. Los porcentajes de sorción fueron de $16,56 \%$ para Cd(II) y 15,6\% para el $\mathrm{Hg}$ (II) en un periodo de 7 días.
\end{abstract}

Palabras clave : Fitorremediación, Eichhornia crassipes, absorción.

\section{STUDY OF PHYSICOCHEMICAL PARAMETERS FOR CADMIUM (II) AND MERCURY (II) PHYTOREMEDIATION USING THE SPECIE Eichhornia crassipes (WATER HYACINTH)}

\begin{abstract}
In this work, the studies were performed to measure the sorption capacity of metal ions Cd (II) and $\mathrm{Hg}$ (II) using the specie Eichhornia crassipes (water hyacinth). This study includes assays where the nutrient concentration, the $\mathrm{pH}$ and the metal ion concentration were optimized. This tests were carried out at room temperature and with aqueous solutions of $\mathrm{Cd}$ (II), $\mathrm{Hg}$ (II), in which the samples of Eichhornia crassipes were placed. To confirm the removal of these metals, the waste solutions after the treatment with the Water Hyacinth species were treated using the method APHA 3030-e. However, Eichhornia crassipes samples were treated using the EPA 200.3 method. The concentration of Cd (II) was determined by a ICP-OES spectrometer and $\mathrm{Hg}$ (II), by an atomic absorption spectrophotometer.

The results showed: Optimal dosage $1 \mathrm{~mL}$ of $\mathrm{A}$ and $0,5 \mathrm{~mL}$ of $\mathrm{B}$, optimum $\mathrm{pH} 5$, optimum concentration of Cd (II) and $\mathrm{Hg}$ (II) $5 \mathrm{mg} / \mathrm{L}$ for each ion. With these parameters, it was started
\end{abstract}

* Laboratorio de investigación en biopolímeros y metalofármacos -LIBIPMET, Facultad de Ciencias.

Universidad Nacional de Ingeniería. Av.Túpac Amaru 210. Rímac, Lima-Perú. vpomal @uni.pe 
the removal of $5 \mathrm{mg} / \mathrm{L}$ of the metal ions contained in $1 \mathrm{~L}$ of solution. Being the percentages of sorption $16,56 \%$ for $\mathrm{Cd}$ (II) and 15,6\% for $\mathrm{Hg}$ (II) after a period of 7 days.

Key words: Phytoremediation, Eichhornia crassipes, absorption.

\section{INTRODUCCIÓN}

En la actualidad existen diversas técnicas propuestas para la rehabilitación de contaminantes (suelo, sedimento, agua, atmósfera, etc.). Estas tecnologías de remediación son muy variables, dependiendo de la matriz contaminada, la naturaleza de los contaminantes, el nivel de contaminación y la disponibilidad de los recursos. El análisis actual del estado de las técnicas de remediación cuenta con un uso cada vez mayor en los países en desarrollo, lo que lleva a la reducción de costos, instalación, operación y monitoreo, debido a que el patrón de la evolución de las tecnologías de remediación se centra en soluciones cada vez menos invasivas. Entre estas nuevas tecnologías que se están investigando, tenemos a la fitorremediación que hace uso de las plantas y sus microorganismos asociados, con el fin de tratar in situ suelos contaminados. Se trata de una tecnología emergente con un gran potencial para la limpieza eficiente y de bajo costo para una amplia gama de contaminantes orgánicos e inorgánicos. La macrófita Eichhornia crassipes (jacinto de agua) es una planta acuática flotante conocida por su capacidad de producción y la eliminación de contaminantes del agua. El jacinto de agua crece en una amplia variedad de tipos de humedales y prefiere aguas con nutrientes enriquecidos. Sin embargo, se puede tolerar una variación considerable en los niveles de nutrientes, temperatura y $\mathrm{pH}$.

El $p \mathrm{H}$ óptimo para el crecimiento de Eichhornia crassipes es de 6-8. Puede crecer en una amplia gama de temperatura de 1 a $40{ }^{\circ} \mathrm{C}$ (óptimo crecimiento entre $25-27,5^{\circ} \mathrm{C}$ ) pero se cree que es sensible al frío. ${ }^{1}$ Las tasas de crecimiento se incrementan con el aumento de las cantidades de nitrógeno y agua. ${ }^{2}$

La técnica de fitorremediación empleada para remover cadmio (II) y mercurio (II) en aguas contaminadas con estos metales, fue aplicada en nuestra investigación, empleando la especie Eichhornia crassipes debido a sus antecedentes de gran capacidad de absorción de estos metales pesados. La determinación de los metales en Eichhornia crassipes y en las soluciones residuales se llevó a cabo mediante técnicas espectrométricas de absorción atómica e ICP óptico, basados en métodos estandarizados, como el método espectrométrico de absorción atómica de vapor frio (APHA 3112-b, 21st ed. 2005), método EPA 200.3: Digestión de metales en tejido por ICPOES/ICPOMS y absorción atómica, método para digestión por ácido nítrico (APHA 3030-e, 21st ed. 2005) y método digestión de metales (APHA3030-d, 21st ed. 2005).

\section{PARTE EXPERIMENTAL}

\section{Muestreo de la especie vegetal Eichhornia crassipes (jacinto de agua):}

La especie vegetal jacinto de agua fue colectada en el valle Chillón, en el distrito de Carabayllo, provincia y departamento de Lima, el 20 de setiembre del 2012. En este lugar crecen en humedales, estanques y piscinas. Luego del muestreo, estas plantas se llevaron a una piscina construida de ladrillo y cemento de dimensiones 1,80 m (largo), 1,20 m (ancho) y $20 \mathrm{~cm}$ (alto), que se utilizó exclusivamente para su almacenamiento y cultivo, las mismas que se llevarían al laboratorio para realizar los ensayos correspondientes. 
En la figura 1, se observa el muestreo de la planta y el agua donde crece el jacinto de agua.

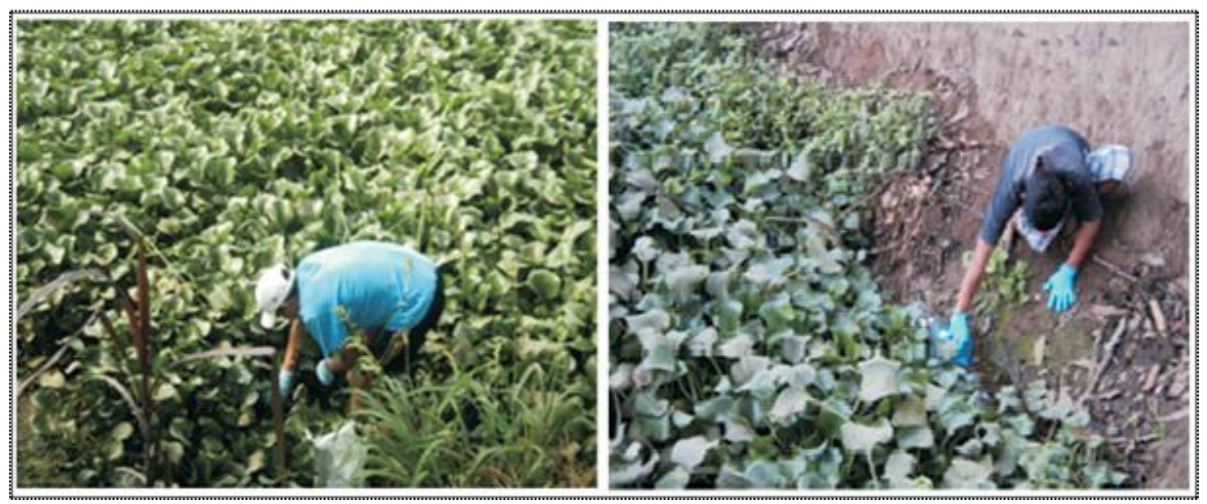

Figura 1: Izquierda (Recolección de jacinto de agua); derecha (Muestreo del agua donde crece el jacinto de agua)

\section{Determinación de la dosis óptima de nutrientes para la viabilidad de Eichhornia} crassipes (jacinto de agua):

Para determinar la dosis óptima de nutrientes se preparó diferentes dosis de solución A y solución B mostrados en la tabla 1, cuya composición química se muestra en la tabla 2, en 1 litro, en las cuales se sometió muestras de un tamaño y peso promedio y se midió el tiempo de supervivencia de cada planta a diferentes dosis de las soluciones A y B. Luego de ser sometidas cada muestra con las diferentes dosis de nutrientes se realizó un seguimiento de cada muestra en función de tiempo (días) y se observó cualitativamente todas las partes de la planta, anotando cualquier diferencia con el aspecto que presenta al inicio.

Efecto del $p H$ sobre la viabilidad de Eichhornia crassipes (jacinto de agua) y la solubilidad del metal:

Para observar el efecto que ocasiona la variación de $p \mathrm{H}$ en el Eichhornia crassipes (jacinto de agua) se preparó diferentes soluciones con la concentración conocida de nutriente ya optimizado, variando el rango de $p \mathrm{H}$ desde 1,5 a 8 y a cada solución se le adicionó 1 ppm de cadmio (II) y 1 ppm de mercurio (II), esto con la finalidad de determinar la solubilidad del ion metálico con respecto al $p \mathrm{H}$ de cada solución, tal como lo muestra la tabla 3 .

Determinación de la concentración óptima de los metales para la viabilidad de Eichhornia crassipes (jacinto de agua):

Con la finalidad de determinar la concentración óptima de metales cadmio (II) y mercurio (II) a la cual la especie Eichhornia crassipes (jacinto de agua) será sometido y soportará el mayor tiempo de vida. Para ello se preparó 1 litro de las diferentes soluciones para cadmio (II) y mercurio (II), a una determinada concentración de nutrientes y $p \mathrm{H}$ ya conocidos. La concentración de cadmio (II), desde 1 a 20 ppm y la del mercurio (II) desde 1 a 25 ppm como se muestra en la tabla 4. Las muestras de jacinto de agua se sometieron simultáneamente a cada una de estas soluciones cuya concentración del metal es conocida 


\section{Determinación de cadmio (II) y mercurio (II) en la misma planta:}

Para este ensayo, se sometieron ocho muestras de Eichhornia crassipes (jacinto de agua) en soluciones con los parámetros necesarios ya optimizados. La recolección se dio cada 24 horas donde se colocaron las muestras en bolsas ziploc y se identificaron como se muestra en la tabla 5, para luego ser digestadas según los métodos establecidos.

\section{Determinación de cadmio (II) y mercurio (II) en las soluciones residuales tratadas con} Eichhornia crassipes (jacinto de agua)

Para determinar las concentraciones en ppm de cadmio (II) y mercurio (II) en las soluciones residuales correspondientes a las muestras de la tabla 4, de las cuales eran conocidas las dosis de nutrientes, el $p \mathrm{H}$ y la concentración inicial de cadmio (II) y mercurio (II). Las muestras sometidas fueron recolectadas cada 24 horas hasta el día ocho; simultáneamente se tomó alícuotas de $50 \mathrm{~mL}$ de solución en digitubos del mismo volumen, los cuales fueron preservados con $1 \mathrm{~mL} \mathrm{HNO}_{3} 1: 1$ y etiquetados como se muestra en la tabla 6. Finalmente digestados con $\mathrm{HNO}_{3}$ concentrado según el método APHA 3030-e. Se escogió a la muestra 5 por duplicado y un blanco reactivo como control. La muestra 5 es representativa porque representa un rango intermedio del tiempo de contacto con la planta (jacinto de agua).

\section{Determinación de cadmio (II) y mercurio (II) independientemente en cada planta}

Para determinar la concentración de cadmio (II) y mercurio (II) independientemente en Eichhornia crassipes (jacinto de agua), se procedió de la misma manera en la que se determinaron ambos metales juntos; un litro de solución y a las mismas condiciones optimizadas; es decir concentración de nutrientes, $p \mathrm{H}$ y concentración del metal. Cada una de las ocho muestras previamente pesadas e identificadas fueron sometidas de manera simultánea a cada recipiente. En la tabla 6 se muestran los pesos de cada muestra (planta) con su respectiva identificación y el peso de muestra que se utilizó para el análisis cada 24 horas hasta el día ocho. La muestra del octavo día se analizó por duplicado, siempre acompañado de una muestra de jacinto de agua como blanco.

\section{Determinación de cadmio (II) y mercurio (II) independientemente en cada solución residual de Eichhornia crassipes}

La concentración de cadmio (II) y mercurio (II) fue determinada en cada solución residual luego de ser removida cada una de las ocho muestras de jacinto de agua. Para cada ion metálico (tabla 7) se tomaron alícuotas de $50 \mathrm{~mL}$ en digitubos, cada una de ellas se preservó con $\mathrm{HNO}_{3}$ 1:1 y se etiquetaron según se muestra en la tabla 8 . Finalmente fueron digestados con ácido nítrico concentrado $\left(\mathrm{HNO}_{3}\right)$ según el método APHA 3030-e. Para todas estas muestras se escogió a la muestra 8 por duplicado y un blanco reactivo como control, luego se procedió a analizar las muestras con el equipo ICP-OES para cadmio (II) y con el equipo de absorción atómica acoplado a un generador de hidruros (vapor frio) para el mercurio(II)

Tabla 1. Dosis de la solución A y B para diferentes muestras (plantas)

\begin{tabular}{|c|c|c|c|c|c|c|c|c|c|c|c|c|}
\hline Muestra $\mathrm{N}^{\circ}$ & 1 & 2 & 3 & 4 & 5 & 6 & 7 & 8 & 9 & 10 & 11 & 12 \\
\hline $\begin{array}{l}\text { Solución "A" } \\
(\mathrm{mL})\end{array}$ & 0,5 & 1 & 1 & 1,5 & 2 & 2 & 2,5 & 2,5 & 5 & 5 & 5 & 10 \\
\hline $\begin{array}{l}\text { Solución "B" } \\
(\mathrm{mL})\end{array}$ & 0,5 & 0,5 & 1 & 1 & 1 & 2 & 1 & 2 & 2 & 2,5 & 5 & 5 \\
\hline pH solución & 5,05 & 5,25 & 5,17 & 5,25 & 5,28 & 5,10 & 5,02 & 5,27 & 5,40 & 5,00 & 4,95 & 5,09 \\
\hline
\end{tabular}


Tabla 2. Composición química de nutrientes

\begin{tabular}{llc}
\hline Tipos de solución & Composición química & Cantidad de cada compuesto \\
\hline \multirow{3}{*}{ Solución } & Nitrato de potasio $\left(\mathrm{KNO}_{3}\right)$ & $110 \mathrm{~g}$ \\
concentrada A & Nitrato de amonio $\left(\mathrm{NH}_{4} \mathrm{NO}_{3}\right)$ & $70 \mathrm{~g}$ \\
& Superfosfato triple $\left[\mathrm{Ca}_{2}\left(\mathrm{H}_{2} \mathrm{PO}_{4}\right)_{2}\right]$ & $36 \mathrm{~g}$ \\
\hline \multirow{2}{*}{ Solución } & Sulfato de magnesio $\left(\mathrm{MgSO}_{4}\right)$ & $110 \mathrm{~g}$ \\
concentrada B & Quelato de hierro $(\mathrm{Fe}-\mathrm{O}, \mathrm{o}-\mathrm{EDDHA})$ & $8,5 \mathrm{~g}$ \\
& Solución de micronutriente & $200 \mathrm{~mL}$ \\
Solución de & Sulfato de manganeso $\left(\mathrm{MnSO}_{4} \cdot 4 \mathrm{H}_{2} \mathrm{O}\right)$ & $5,0 \mathrm{~g}$ \\
micronutrientes & Acido bórico $\left(\mathrm{H}_{3} \mathrm{BO}_{3}\right)$ & $3,0 \mathrm{~g}$ \\
& Sulfato de zinc $\left(\mathrm{ZnSO}_{4} \cdot 7 \mathrm{H}_{2} \mathrm{O}\right)$ & $1,7 \mathrm{~g}$ \\
& Sulfato de cobre $\left(\mathrm{CuSO}_{4} \cdot 5 \mathrm{H}_{2} \mathrm{O}\right)$ & $1,0 \mathrm{~g}$ \\
\hline
\end{tabular}

Tabla 3. Muestras de Eichhornia crassipes (jacinto de agua) para la optimización del $p \mathrm{H}$ y la solubilidad de cadmio (II) y mercurio (II).

\begin{tabular}{cccc}
\hline $\begin{array}{c}\text { Identificación de } \\
\text { soluciones }\end{array}$ & $\begin{array}{c}\text { Concentración de Cd (II) } \\
(\mathbf{p p m})\end{array}$ & $\begin{array}{c}\text { Concentración de Hg (II) } \\
(\mathbf{p p m})\end{array}$ & $\begin{array}{c}p \text { H de } \\
\text { solución }\end{array}$ \\
\hline $\mathbf{1}$ & 1 & 1 & 1,5 \\
$\mathbf{2}$ & 1 & 1 & 2 \\
$\mathbf{3}$ & 1 & 1 & 3 \\
$\mathbf{4}$ & 1 & 1 & 4 \\
$\mathbf{5}$ & 1 & 1 & 5 \\
$\mathbf{6}$ & 1 & 1 & 6 \\
$\mathbf{7}$ & 1 & 1 & 7 \\
$\mathbf{8}$ & 1 & 1 & 8 \\
\hline
\end{tabular}

Tabla 4. Número de muestras de Eichhornia crassipes (jacinto de agua) sometidas a una determinada concentración de cadmio (II) y mercurio (II).

\begin{tabular}{ccc}
\hline $\begin{array}{c}\text { Numero de muestras de } \\
\text { Jacinto de agua }\end{array}$ & \multicolumn{2}{c}{ Concentración del ion metálicos (ppm) } \\
\cline { 2 - 3 } & $\mathbf{C d}($ II) & Hg (II) \\
\hline 1 & 1 & 1 \\
1 & 5 & 5 \\
1 & 10 & 10 \\
1 & 15 & 15 \\
1 & 20 & 20 \\
1 & - & 25 \\
\hline
\end{tabular}


Tabla 5. Identificación y pesos de las muestras (plantas) recolectadas.

\begin{tabular}{cccc}
\hline $\begin{array}{c}\text { Identificación de las } \\
\text { muestras P=Plantas }\end{array}$ & $\begin{array}{c}\text { Tiempo de } \\
\text { recolección (Horas) }\end{array}$ & $\begin{array}{c}\text { Peso de cada } \\
\text { planta (g) }\end{array}$ & $\begin{array}{c}\text { Peso húmedo de } \\
\text { muestras (g) }\end{array}$ \\
\hline M-1-D(P) & 24 & 27,65 & 5,0020 \\
M-2-D(P) & 48 & 27,04 & 5,0050 \\
M-3-D(P) & 72 & 27,49 & 5,0020 \\
M-4-D(P) & 96 & 26,15 & 5,0030 \\
M-5-D(P) & 120 & 27,60 & 5,0060 \\
M-5-D-Dup(P) & 120 & 27,55 & 5,0011 \\
M-6-D(P) & 144 & 27,75 & 5,0021 \\
M-7-D(P) & 168 & 26,12 & 5,0040 \\
M-8-D(P) & 192 & 26,62 & 5,0015 \\
BK & 192 & 28,51 & 5,0010 \\
\hline
\end{tabular}

Nota: M-1-D(P) corresponde a la muestra de planta tomada en un día.

Tabla 6. Identificación de las muestras recolectadas para cada tiempo

\begin{tabular}{cccc}
\hline $\begin{array}{c}\text { Identificación de las muestras } \\
\text { SR=solución residual }\end{array}$ & $\begin{array}{c}\text { Tiempo de } \\
\text { recolección (horas) }\end{array}$ & $\begin{array}{c}\text { Volumen de muestra } \\
(\mathbf{m L})\end{array}$ & $\begin{array}{c}\text { Preservante } \\
\text { HNO }_{\mathbf{3}} \mathbf{1 : 1}(\mathbf{m L})\end{array}$ \\
\hline M-1-D(SR) & 24 & 50 & 1 \\
M-2-D(SR) & 48 & 50 & 1 \\
M-3-D(SR) & 72 & 50 & 1 \\
M-4-D(SR) & 96 & 50 & 1 \\
M-5-D(SR) & 120 & 50 & 1 \\
M-5-D-DUP(SR) & 120 & 50 & 1 \\
M-6-D(SR) & 144 & 50 & 1 \\
M-7-D(SR) & 168 & 50 & 1 \\
M-8-D(SR) & 192 & 50 & 1 \\
BK & 192 & 50 & \\
\hline
\end{tabular}

Nota: M-1-D(SR) corresponde a la muestra de solución residual tomada en un día.

Tabla 7. Identificación y pesos de las muestras recolectadas para cada tiempo.

\begin{tabular}{ccccccc}
\hline $\begin{array}{c}\text { Tiempo de } \\
\text { recolección para } \\
\text { cada muestra } \\
\text { (horas) }\end{array}$ & $\begin{array}{c}\text { Identificación de } \\
\text { las muestras } \\
\mathbf{p = p l a n t a}\end{array}$ & $\begin{array}{c}\text { Peso de } \\
\text { cada } \\
\text { planta }(\mathbf{g})\end{array}$ & $\begin{array}{c}\text { Peso húmedo } \\
\text { de muestras } \\
(\mathbf{g})\end{array}$ & $\begin{array}{c}\text { Identificación de } \\
\text { las muestras } \\
\text { p=planta }\end{array}$ & $\begin{array}{c}\text { Peso de } \\
\text { cada } \\
\text { planta (g) }\end{array}$ & $\begin{array}{c}\text { Peso húmedo } \\
\text { de muestras } \\
(\mathbf{g})\end{array}$ \\
\hline 24 & M-1-D-Cd(P) & 55,25 & 4,9990 & M-1-D-Hg(P) & 56,44 & 5,0010 \\
48 & M-2-D-Cd (P) & 55,23 & 4,9960 & M-2-D- Hg(P) & 56,34 & 5,0260 \\
72 & M-3-D-Cd (P) & 53,63 & 4,9900 & M-3-D- Hg(P) & 57,75 & 5,0270 \\
96 & M-4-D-Cd (P) & 56,32 & 4,9980 & M-4-D- Hg(P) & 52,95 & 5,0030 \\
120 & M-5-D-Cd (P) & 56,45 & 4,9750 & M-5-D- Hg(P) & 54,70 & 5,0030 \\
120 & M-6-D-Cd(P) & 55,51 & 5,0050 & M-6-D- Hg(P) & 55,30 & 5,0140 \\
144 & M-7-D-Cd (P) & 56,7 & 5,0040 & M-7-D- Hg(P) & 55,17 & 5,0010 \\
168 & M-8-D-Cd (P) & 55,49 & 5,0010 & M-8-D- Hg(P) & 55,58 & 5,0020 \\
192 & M-8-D-Dup-Cd (P) & 55,45 & 5,0015 & M-8-D-Dup-Hg(P) & 55,60 & 5,0024 \\
192 & BK & 51,88 & 5,0366 & BK & 58,42 & 5,0770 \\
\cline { 2 - 7 }
\end{tabular}


Tabla 8. Identificación de las soluciones residuales de cadmio (II) y mercurio (II) recolectadas para cada tiempo

\begin{tabular}{ccccc}
\hline $\begin{array}{c}\text { Volumen de muestra } \\
\text { recolectada con 1mL de } \\
\text { Preservante } \\
\mathbf{H N O}_{\mathbf{3}} \mathbf{1 : 1}(\mathbf{m L})\end{array}$ & $\begin{array}{c}\text { Identificación de las } \\
\text { muestras }\end{array}$ & $\begin{array}{c}\text { Tiempo de } \\
\text { recolección } \\
\text { (horas) }\end{array}$ & $\begin{array}{c}\text { Identificación de las } \\
\text { muestras } \\
\text { SR=Solución } \\
\text { residual }\end{array}$ & $\begin{array}{c}\text { Tiempo de } \\
\text { recolección } \\
\text { (horas) }\end{array}$ \\
\hline & M-1-D-Cd(SR) & 24 & M-1-D-Hg(SR) & 24 \\
& M-2-D-Cd(SR) & 48 & M-2-D-Hg(SR) & 48 \\
M-3-D-Cd(SR) & 72 & M-3-D-Hg(SR) & 72 \\
M-4-D-Cd(SR) & 96 & M-4-D-Hg(SR) & 96 \\
& M-5-D-Cd(SR) & 120 & M-5-D-Hg(SR) & 120 \\
$50 \mathrm{~mL}$ & M-6-D-Cd(SR) & 120 & M-6-D-Hg(SR) & 120 \\
& M-7-D-Cd(SR) & 144 & M-7-D-Hg(SR) & 144 \\
& M-8-D-Cd(SR) & 168 & M-8-D-Hg(SR) & 168 \\
& M-8-D-Dup-Cd (SR) & 192 & M-8-D-Dup-Hg(SR) & 192 \\
& BK & 192 & BK & 192 \\
\hline
\end{tabular}

\section{RESULTADOS Y DISCUSIÓN}

\section{Determinación de la dosis óptima de nutrientes para la viabilidad de Eichhornia crassipes (jacinto de agua)}

Luego que las muestras de jacinto de agua fueron sometidas a diferentes dosis de nutrientes durante varios días en el laboratorio a temperatura de $25 \pm 2{ }^{\circ} \mathrm{C}$, se observó que algunas de estas se secaban, morían y otras crecían con nuevas hojas y tallos. Luego de 25 días la muestra con dosis $\mathrm{A}=1 \mathrm{~mL}$ y $\mathrm{B}=0,5 \mathrm{~mL}$ se mantuvo con hojas y tallos verdes, desarrollando algunas hojas en tamaño, gracias a los nutrientes que aprovechó y a partir de este tiempo comenzó a secar y perder tamaño lentamente, por falta de nutrientes hasta el día 28 , siendo esta dosis la óptima para nuestros objetivos.

Efecto del $p H$ sobre la viabilidad de Eichhornia crassipes (jacinto de agua) y la solubilidad del metal.

Los iones cadmio (II) y mercurio (II) empiezan a precipitar como hidróxidos en presencia de los nutrientes alrededor de $p \mathrm{H}>7$. Combinando ambos aspectos, se dedujo que $p \mathrm{H} 5$ es el valor óptimo porque mantiene viva a la planta por más de 18 días para el cadmio (II) y 20 días para el mercurio (II). Estudios realizados determinaron el rol del $p \mathrm{H}$ en un proceso similar, encontrando que el $p \mathrm{H}$ ejerce un fuerte efecto: (i) en la formación de iones complejos del metal en solución acuosa, los cuales presentan diferentes ligandos que modifican la acidez y morfología del metal en solución, y (ii) en el estado químico de los centros activos de los adsorbentes. ${ }^{3,4}$ Asimismo, debemos tener en cuenta que la solubilidad del cadmio (II) y mercurio (II) también es afectada por la presencia de diversos aniones quelantes provenientes de las soluciones nutrientes (por ejemplo fosfatos, nitratos y sulfatos), tornando más complejo aún este fenómeno.

Determinación de la concentración óptima de los metales para la viabilidad de las plantas

Se determinó la concentración de cadmio (II) y mercurio (II) a la cual la planta presenta fitotoxicidad. A una concentración de $5 \mathrm{mg} / \mathrm{L}$, la planta permanece viva por tiempo suficiente para cumplir su tarea destoxificante. No todas las plantas son capaces de resistir altas concentraciones de metales pesados, Eichhornia crassipes (jacinto de agua), es una de las 
pocas especies capaces de acumular metales tóxicos, hasta ciertos límites, sin alterar su metabolismo. Se piensa que el reemplazo de cationes divalentes como magnesio y calcio intracelular por metales pesados isovalentes como cobre, plomo y cadmio causa desequilibrio en los sistemas vivos al insertarse en los sistemas enzimáticos de forma irreversible.

Determinación de la concentración de cadmio (II) y mercurio (II) presentes en Eichhornia crassipes (jacinto de agua) y en las soluciones residuales.

Estos metales pesados, Cd (II) y Hg (II), son no esenciales, ni cumplen una función fisiológica conocida en plantas. Otros elementos como $\mathrm{Co}, \mathrm{Cu}, \mathrm{Fe}, \mathrm{Mn}, \mathrm{Mo}, \mathrm{Ni}$ y $\mathrm{Zn}$, son esenciales para el crecimiento y el metabolismo normal de las plantas.

Los metales pesados como Cd (II) y Hg (II) son altamente tóxicos, debido a su reactividad con los átomos de $\mathrm{S}$ y $\mathrm{N}$ presentes en los aminoácidos y las proteínas. ${ }^{6}$ Contrario a los contaminantes de origen orgánico, estos metales pesados no pueden ser degradados química u orgánicamente, por lo que una de las alternativas para la remoción de aguas contaminadas con estos iones metálicos es la fitoextracción. Ésta consiste en la absorción y acumulación de metales pesados en la parte aérea de plantas hiperacumuladoras como Eichhornia crassipes. Esta planta puede presentar diferentes mecanismos de tolerancia en respuesta al exceso de $\mathrm{Cd}$ (II) y $\mathrm{Hg}$ (II), incluida la reducción del transporte a través de membrana de exclusión, formación de péptidos ricos en grupos tiólicos (fitoquelatinas y metalotioneínas), quelatación por ácidos orgánicos, aminoácidos y compartimentación del metal en estructuras subcelulares.

La acumulación de los iones metálicos Cd (II) y $\mathrm{Hg}$ (II) por Eichhornia crassipes (jacinto de agua) se da probablemente en un proceso complejo, desarrollado en varias etapas: a) bioactivación del ion metálico en la rizosfera, b) transporte del ion metálico a través de la membrana plasmática de las células radiculares, c) traslocación del ion metálico a través del xilema, d) destoxificación y secuestro del ion metálico por la planta. ${ }^{7}$

Luego de ser recolectadas las muestras de jacinto de agua, se las llevó a una digestión según el método EPA 200.3 con $\mathrm{HNO}_{3}-\mathrm{H}_{2} \mathrm{O}_{2}$ y el digestado fue enrasado a un volumen final de $50 \mathrm{~mL}$ con agua ultrapura. Se procedió a la lectura de estas muestras con el equipo de ICP-OES para el cadmio y la absorción atómica (vapor frío) para el mercurio (II). Se realizó los ensayos por duplicado de la muestra correspondiente al quinto día.
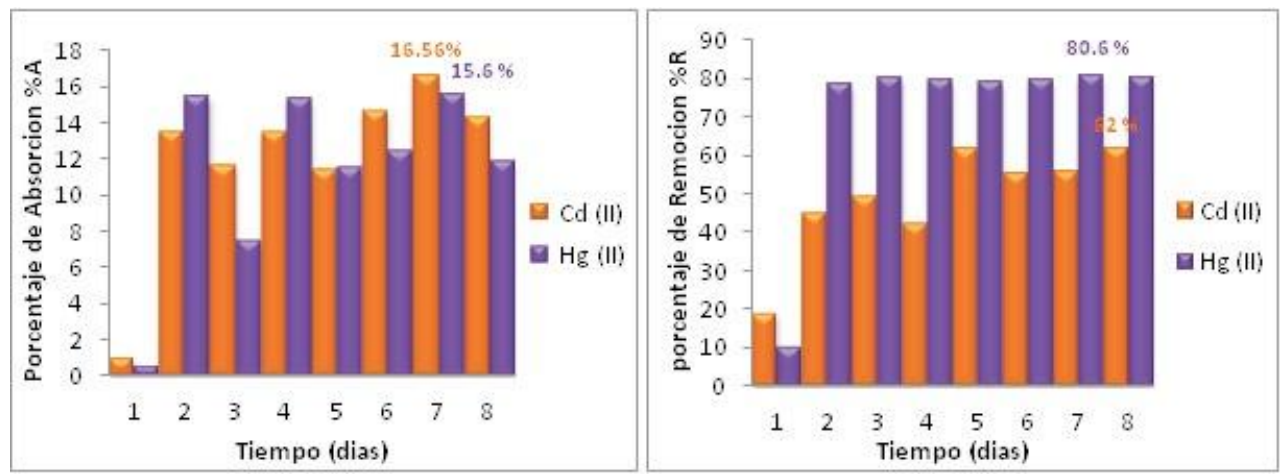

Figura 2. Izquierda; porcentaje de absorción de Cd (II) y Hg (II) en Eichhornia crassipes. Derecha; porcentaje de remoción de Cd (II) y Hg (II) en las soluciones residuales. Estas muestras se sometieron a una solución conocida de $5 \mathrm{mg} / \mathrm{L}$ de Cd(II) y Hg (II) en una misma solución durante ocho días. 
En la figura 2 se muestra una ligera diferencia entre el porcentaje de absorción de estos iones lo cual se debe probablemente a la estabilización de los complejos de cadmio (II) con respecto a los complejos formados con el mercurio (II) y que guarda relación con las constantes de estabilidad de cadmio (II) y mercurio (II) frente a un ligando tiolato, siendo los valores $\log _{\mathrm{Cd}}$ $=5,6$ y $\operatorname{LogK}_{\mathrm{Hg}}=10,2$ a un $p \mathrm{H}$ 7. Estos valores muestran que hay una mayor estabilidad por parte del mercurio (II); sin embargo, se debe tener en cuenta otros factores, como la solubilidad de la especie que libera el ion metálico, la energía de enlace entre el ion metálico y el azufre (MS) y la accesibilidad del metal al nucleófilo, siendo este último factor más favorable para el Cd (II) debido a su número de coordinación 4 (tetraédrico) lo cual explica porque absorbe un poco más que el Hg (II), siendo ambos muy afines a los complejos que contiene la planta.

En las figuras 3 y 4 se observa los máximos porcentajes de absorción y remoción. Siendo $6,41 \%$ en un periodo de siete días y un porcentaje de remoción de $63,4 \%$ en un periodo de tres días para el cadmio (II). Mientras que se observa el porcentaje de absorción de 15,23\% en un periodo de un día y un porcentaje de remoción de $91,8 \%$ en un periodo de ocho días para el mercurio (II).

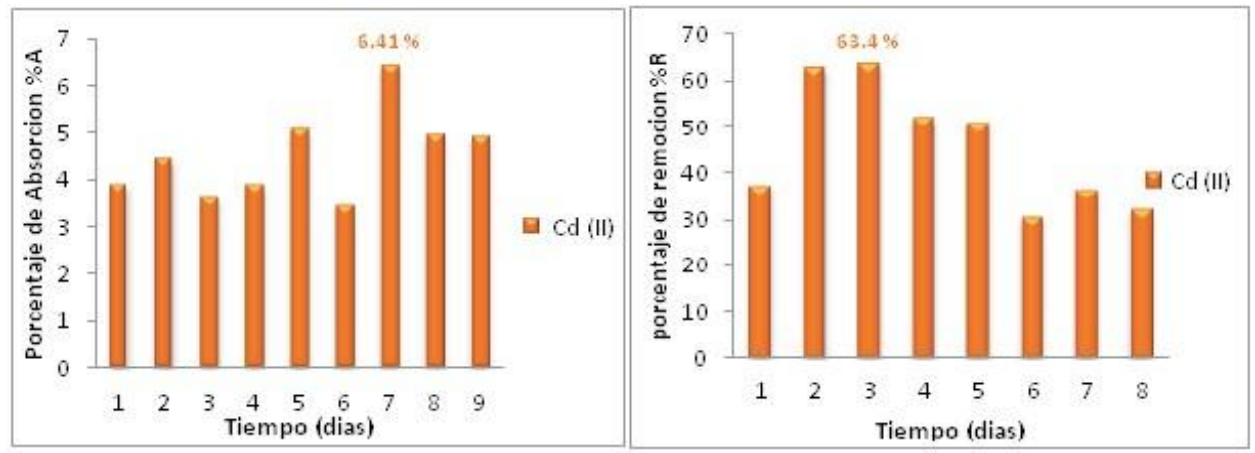

Figura 3. Izquierda; porcentaje de absorción de Cd (II) en Eichhornia crassipes. Derecha; porcentaje de remoción de Cd (II) en las soluciones residuales. Estas muestras se sometieron en soluciones de $5 \mathrm{mg} / \mathrm{L}$ de Cd (II) libre de $\mathrm{Hg}$ (II), para un periodo de pcho días.
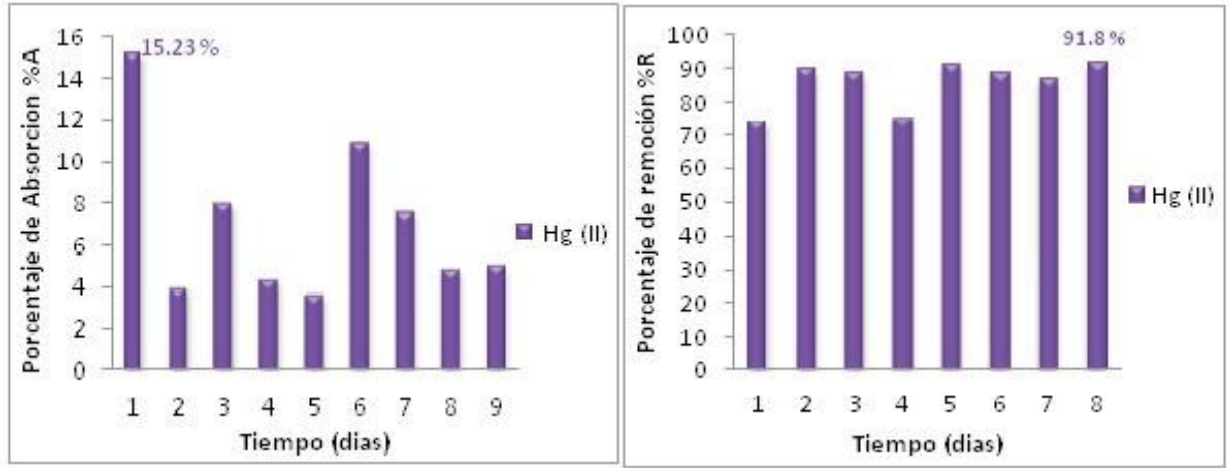

Figura 4. Izquierda; porcentaje de absorción de Hg (II) en Eichhornia crassipes. Derecha; porcentaje de remoción de $\mathrm{Hg}$ (II) en las soluciones residuales. Estas muestras se sometieron en soluciones de $5 \mathrm{mg} / \mathrm{L}$ de $\mathrm{Hg}$ (II) libre de Cd (II). 


\section{CONCLUSIONES}

Se logró determinar las condiciones óptimas para obtener una absorción cuantitativa de $\mathrm{Cd}$ (II) y Hg (II) con Eichhornia crassipes (jacinto de agua), siendo los resultados obtenidos, dosis óptima $1 \mathrm{~mL}$ de $\mathrm{A}$ y $0,5 \mathrm{~mL}$ de $\mathrm{B}, p \mathrm{H} 5$, concentración de $\mathrm{Cd}$ (II) y $\mathrm{Hg}$ (II) $5 \mathrm{mg} / \mathrm{L}$ y porcentaje de absorción máxima en Eichhornia crassipes, 16,56 \% para el Cd (II) y 15,60 \% para el $\mathrm{Hg}$ (II) en el sétimo día, demostrando así la efectividad de la técnica de fitorremediación.

Por lo tanto, la especie Eichhornia crassipes (jacinto de agua) es una planta potencialmente aprovechable para remover cadmio (II) y mercurio (II) en aguas contaminadas; dándole las condiciones óptimas para realizar el proceso se obtienen resultados favorables para la remoción de estos iones metálicos estudiados.

\section{AGRADECIMIENTOS}

Agradezco, en primer lugar, a mi familia por su paciencia en el desarrollo de esta tesis de investigación, por su apoyo desinteresado durante el periodo que demandó este trabajo.

De igual forma, agradezco al técnico químico Julio Trauco, por su colaboración en la lectura de las concentraciones de los iones cadmio (II) y mercurio (II) en las muestras de Eichhornia crassipes, mediante la técnica de absorción atómica e ICP-OES.

\section{BIBLIOGRAFÍA}

1. Wilson J.R., Holst N., Rees M. Determinantes y los patrones de crecimiento de la población en Jacinto de agua. Aquat Bot 2005; 81:51-67.

2. Heard T.A., Winterton S.L. Las interacciones entre el estado nutricional y el picudo herbivoria en el control biológico del jacinto de agua. J.Appl.Ecol 2000; 37:117-127.

3. Blanco D., Llanos B., Cuizano N.A., Maldonado H. y Navarro A.E. Optimización de la Adsorción de cadmio divalente en Lessonia trabeculata mediante reticulación de $\mathrm{CaCl}_{2}$. Revista de la Sociedad Química del Perú 2005; 71:237-245.

4. Navarro A.E., Ramos K.P., Campos K. y Maldonado H. Elucidación del Efecto del pH en la adsorción de metales pesados mediante biopolímeros naturales: Cationes Divalentes Superficies Activas. Revista Iberoamericana de Polimeros 2006 ; 7(2):115-128.

5. Salt, D.E.; Rauser, W.E. Mg ATP-dependent transport of phytochelatins across the tonoplast of oat roots. Plant Physiol. 1995;107: 1293-1301.

6. Clemens, S. Molecular mechanisms of plant metal tolerance and homeostasis. Planta 2001;212:475- 486.

7. Yang X., Feng, Y., He Z., Stoffella, P.J. Molecular mechanisms of heavy metal hyperaccumulation and phytoremediation. Journal of Trace Elements in Medicine and Biology 2005; 18:339-353. 